\title{
¿Puede el sector informal afectar a la relación entre desempleo y producción? Un análisis del caso de México
}

\author{
Alejandro Islas Camargo y Willy W. Cortez ${ }^{1}$
}

\section{Resumen}

Un aspecto fundamental de los países en desarrollo es la existencia de un gran sector informal. En el presente trabajo se analiza el efecto que este rasgo tiene sobre la relación entre las variaciones en el desempleo y el crecimiento de la producción en el caso de México, un país que se caracteriza por la existencia de un gran sector informal. Partiendo de estudios recientes sobre el coeficiente de Okun, en primer lugar, pondremos a prueba si la relación ente los componentes cíclicos de desempleo y producción es asimétrica. A continuación, exploraremos la posibilidad de que esta relación no lineal pueda verse afectada por variaciones en el sector informal. Nuestros resultados apuntan a la existencia de una relación asimétrica entre los componentes cíclicos.

\section{Palabras clave}

Sector informal, movilidad de la mano de obra, desempleo, crecimiento económico, modelos matemáticos, estudios de casos, México

\section{Clasificación JEL}

C34, E23, E24, E32

\section{Autores}

Alejandro Islas Camargo es Profesor de Tiempo Completo en el Departamento Académico de Estadística del Instituto Tecnológico Autónomo de México (ITAM). Correo electrónico: aislas@itam.mx.

Willy W. Cortez es Profesor e Investigador titular en el Departamento de Métodos Cuantitativos del Centro Universitario de Ciencias Económico Administrativas (CUCEA) de la Universidad de Guadalajara (México). Correo electrónico: wcortez@cucea.udg.mx.

\footnotetext{
Los autores reconocen y agradecen las aportaciones de los participantes en el Seminario de Investigación del Centro Universitario de Ciencias Económico Administrativas (CUCEA) y de Jaime Ros. Cualquier error presente es responsabilidad exclusiva de los autores. Alejandro Islas Camargo quisiera reconocer el apoyo brindado por la Asociación Cultural Mexicana y por el Consejo Nacional de Ciencia y Tecnología (CONACYT) a través de su programa de becas sabáticas. El presente estudio fue realizado mientras Alejandro Islas Camargo visitaba el Departamento de Economía, el Nepal Study Center y el Centro de Política Sanitaria Robert Wood Johnson Foundation (RWJF) en la Universidad de Nuevo México (Estados Unidos).
} 


\section{Introducción}

Una de las relaciones centrales de la macroeconomía, conocida como ley de Okun, determina el costo del desempleo en términos de producción². A partir del importante artículo de Okun (1962), en los primeros estudios se encontró que una alteración de 1 punto porcentual en el desempleo provocaría una alteración de alrededor de 3 puntos porcentuales en la producción en el sentido opuesto. Ahora sabemos que el coeficiente de Okun no permanece constante por una serie de motivos, como por ejemplo cambios en las instituciones del mercado laboral y cambios tecnológicos y demográficos ${ }^{3}$.

En un estudio reciente sobre un grupo de 71 países desarrollados y en desarrollo, Ball y otros (2016) llegan a la conclusión de que el desempleo es menos sensible a las fluctuaciones de la producción en los países en desarrollo que en los países desarrollados. En concreto, el coeficiente de Okun es, en promedio, en torno a un 50\% menor en las economías en desarrollo que en las economías desarrolladas. Estos autores destacan, además, que en la literatura se han identificado diversos factores que pueden ayudar a explicar la diferencia entre estos dos tipos de economías por lo que respecta a la capacidad de respuesta del desempleo ante las fluctuaciones de la producción.

Uno de estos factores es el tamaño de la economía sumergida o sector informal ${ }^{4}$. En su análisis de corte transversal, estos autores encuentran algunas pruebas de que la existencia de un sector informal afecta a la relación entre el mercado laboral formal y la producción medida: cuanto mayor sea el sector informal, menor será el coeficiente de Okun. Dell'Anno y Solomon (2008) concluyeron que el sector informal reducía la magnitud del coeficiente de Okun incluso en el caso de grandes economías como la de los Estados Unidos. Sin embargo, un inconveniente de su estudio es que no se aportó una explicación acerca del mecanismo mediante el cual el sector informal pudiera afectar a este coeficiente. Otro defecto importante es que no se consideró la posibilidad de que el coeficiente pudiera cambiar con el tiempo, ni de que la relación entre desempleo y producción pudiera cambiar a lo largo del ciclo económico. Todavía no se conoce el mecanismo mediante el cual el sector informal afecta al funcionamiento de los mercados laborales en economías desarrolladas y, en especial, en economías en desarrollo. Estas últimas constituyen el objeto del presente estudio. Así pues, es importante analizar la relación entre desempleo abierto y empleo informal, así como la relación de ambos con las fluctuaciones de la producción. Se espera que este análisis ayude a comprender mejor las dinámicas del mercado laboral en los países menos desarrollados, en especial su desempeño en materia de desempleo.

Es bien sabido que un rasgo primordial de los mercados laborales en los países menos desarrollados es la existencia de un gran sector informal. De acuerdo con la Organización Internacional del Trabajo (OIT), más de la mitad del empleo no agrícola en los países en desarrollo se da en el sector informal. En América Latina, por ejemplo, la OIT estima que el empleo informal oscila desde el 30,9\% de los trabajadores empleados en Costa Rica hasta aproximadamente un 72,8\% en Honduras y un 73,6\% en Guatemala. En México, aproximadamente el 53,8\% del empleo no agrícola es informal.

La OIT asocia el empleo informal a malas condiciones laborales y un aumento de la pobreza 5 . La informalidad se ha asociado también a otras de las características de las economías en desarrollo: elevada evasión fiscal, cobertura limitada de las instituciones de seguridad social y reducida capacidad para manejar la economía (Vuletin, 2008).

\footnotetext{
2 O el impacto del crecimiento de la producción sobre las variaciones en el desempleo.

3 Véase una breve reseña sobre estos estudios en Silvapulle, Moosa y Silvapulle (2004) e Islas y Cortez (2013), entre otros.

4 Los demás son la tasa media de desempleo, el PIB per cápita, la participación del sector servicios en el PIB y la utilización inadecuada de las competencias (Ball y otros, 2016).

5 Véase [en línea] http://www.ilo.org/global/topics/employment-promotion/informal-economy/lang--es/index.htm.
} 
En el presente artículo argumentamos no solo que el empleo informal reduce la sensibilidad del desempleo a las variaciones en la producción, sino que además puede ayudar a comprender la dinámica del mercado laboral en los países menos desarrollados. Proponemos un modelo empírico en el que la transición de un régimen a otro depende de la variación en el empleo informal. A partir de aportaciones recientes sobre la no linealidad del coeficiente de Okun, se proponen tres modelos: un modelo lineal, un modelo no lineal con probabilidades de transición fijas (PTF) y un modelo con probabilidades de transición variables en el tiempo (PTV).

Existen distintos modelos teóricos que analizan los efectos macroeconómicos del sector informal en las economías en desarrollo. Por ejemplo, Agénor y Aizenman (1999) concluyen que, dentro de un marco de equilibrio general, no existe una relación estrecha entre las variaciones en la producción y el desempleo, y que un choque macroeconómico negativo desplaza a los trabajadores empleados en el sector formal hacia el sector informal, con un efecto muy limitado sobre la tasa de desempleo agregada. Destaca también el estudio de Bhattacharya (2007), en el que se analiza la relación entre el sector informal y la distribución de ingresos en los países menos desarrollados, así como el de Goldberg y Pavcnick (2003), en el que se estudia la relación entre liberalización comercial e informalidad. La principal aportación del presente estudio no pretende ser teórica, sino que su objetivo es más bien proporcionar una explicación verosímil de las dinámicas del mercado laboral cuando existe un sector informal de gran magnitud.

México es una de las economías más grandes de América Latina, con un importante sector informal no agrícola. También representa un estudio de caso único, porque hace aproximadamente tres décadas que muestra una de las tasas de desempleo más bajas de la región, al tiempo que su desempeño en materia de crecimiento ha venido siendo decepcionante. Estas son las razones que nos hicieron escoger México para nuestro estudio.

El presente artículo se organiza en seis secciones más: en la sección II se examinan estudios recientes sobre movilidad de la mano de obra entre los sectores formal e informal en algunos países de América Latina. En la sección III se revisan brevemente algunos trabajos sobre la ley de Okun. A continuación, en la sección IV se consideran las últimas estimaciones del coeficiente de Okun para México y se describe el desempeño del sector informal en el período de análisis. Un bajo crecimiento es compatible con una baja tasa de desempleo cuando existe un sector informal de gran magnitud. En la sección $V$ se describen los modelos econométricos utilizados para estimar la relación existente entre los componentes cíclicos de la producción y el desempleo. Se proponen tres modelos y se pone a prueba si la relación entre el desempleo y la producción es no lineal. En esta sección se describen también los datos utilizados en el estudio. El análisis empírico se presenta en la sección VI, mientras que en la sección VII se presentan algunas conclusiones.

\section{El sector informal y la movilidad de la mano de obra en América Latina}

Un tema clave en la literatura especializada es la definición de informalidad. Se trata de un concepto que ha cambiado con el paso de los años. Originariamente, este término se utilizaba para describir una situación en la que se empleaba a trabajadores pobres en pequeñas unidades de producción como consecuencia de una falta de mejores oportunidades de empleo. Más adelante, este concepto se amplió para incluir a todos los trabajadores que no estaban cubiertos por la legislación laboral o la seguridad social (Tokman, 2011). Hoy en día el concepto es más complejo, ya que incluye una amplia variedad de actividades del mercado laboral que se pueden agrupar a grandes rasgos en dos grupos principales: por un lado, las actividades de supervivencia (trabajos ocasionales, trabajos 
temporales, trabajos no remunerados, agricultura de subsistencia, pluriempleo) y, por otro, aquellas actividades elegidas racionalmente que se llevan a cabo para evadir impuestos, la legislación laboral u otras normas gubernamentales o institucionales. Aquí se incluyen las empresas no inscritas ante las instituciones reguladoras 6 .

No obstante, el consenso sobre la definición de informalidad no se ha traducido en un consenso sobre la forma de medirla. Algunos investigadores han considerado a los trabajadores empleados en pequeñas unidades de producción no inscritas ante las instituciones reguladoras como medida aproximada de las empresas informales, mientras que otros han preferido tomar como referencia a los trabajadores sin beneficios sociales (por ejemplo, seguro médico y vacaciones pagadas). Un tercer grupo de investigadores combina ambas definiciones. Así, en el estudio de Maloney (1998) sobre el mercado laboral mexicano se definen tres tipos de trabajadores informales: i) trabajadores por cuenta propia, como propietarios de empresas informales con o sin otros empleados; ii) asalariados informales, empleados en empresas informales, y iii) trabajadores subcontratados (aquellos que no reciben un salario regular, sino que cobran un porcentaje o una cantidad a destajo, por comisión o con un contrato por tiempo definido, y que a menudo están en relación con empresas más grandes). Gong, van Soest y Villagómez (2004) consideran a los trabajadores empleados en pequeñas unidades de producción (de menos de cinco trabajadores), mientras que, en el estudio de Calderón (2000) sobre el mercado laboral mexicano, se considera informales a aquellos trabajadores que no están inscritos en las instituciones de seguridad social. Bosch y Maloney (2010) incluyen tanto a trabajadores de pequeñas empresas como a los que no están cubiertos por la normativa laboral. Galli y Kucera (2003) emplean una definición ligeramente distinta: además de los empleados de empresas pequeñas, incluyen a trabajadores por cuenta propia y empleados domésticos.

Existen dos cuestiones importantes que los investigadores han abordado en discusiones recientes sobre los mercados laborales de las economías desarrolladas y en desarrollo: la primera es si se puede considerar que estos mercados están segmentados o no y la segunda, en qué medida son flexibles para ajustarse a un choque en la producción. Por ejemplo, Maloney (1998) aporta datos empíricos en contra de la hipótesis del mercado laboral segmentado en el caso de México; para ello, estima las probabilidades de transición entre los sectores formal e informal. En un análisis longitudinal de tres cohortes de trabajadores ${ }^{7}$, encuentra una probabilidad significativa de transición desde los distintos tipos de informalidad hacia un empleo formal y viceversa. Estima que la probabilidad de transición desde un trabajo informal como asalariado, subcontratado y trabajador por cuenta propia hacia un trabajo formal como asalariado se sitúa en torno al $42 \%$, mientras que las probabilidades de transición en sentido contrario son de un $6 \%$, un $4 \%$ y un $5 \%$, respectivamente. Es decir, existe un flujo bidireccional, pero el movimiento desde el empleo informal hacia el empleo formal es muy superior al movimiento del empleo formal al informal ${ }^{8}$.

Galli y Kucera (2003), por su parte, argumentan desde una hipótesis de amortiguamiento según la cual el empleo informal es anticíclico, es decir, que se puede esperar que un choque negativo en la producción se traduzca en una expansión del sector informal. No obstante, no se puede esperar que todos los distintos tipos de empleo informal respondan del mismo modo a los ciclos económicos. Por ejemplo, es probable que la informalidad motivada por una estrategia de subsistencia sea anticíclica, mientras que el trabajo voluntario por cuenta propia será procíclico. Del mismo modo, el empleo

6 Véase Grupo Banco Mundial [en línea] http://lnweb90.worldbank.org/eca/eca.nsf/1f3aa35cab9dea4f85256a77004e4ef4/2e4 ede543787a0c085256a940073f4e4?OpenDocument.

7 Se tomaron tres cohortes de trabajadores de la Encuesta Nacional de Empleo Urbano (ENEU) en el período comprendido entre el tercer trimestre de 1990 y el segundo trimestre de 1992. Las cohortes son: tercer trimestre de 1990 a tercer trimestre de 1991, primer trimestre de 1991 a primer trimestre de 1992 y segundo trimestre de 1991 a segundo trimestre de 1992.

8 El análisis abarca a hombres trabajadores de entre 16 y 65 años, con una educación de nivel secundario o inferior, en 16 áreas metropolitanas. Se utilizan dos definiciones de empleo informal: trabajadores desprotegidos y personas que trabajan en microempresas. 
informal será procíclico si las empresas deciden aumentar sus subcontrataciones durante fases de expansión, pero será anticíclico si deciden reducir este tipo de empleo en dichas fases.

Bosch y Maloney (2010), por ejemplo, encuentran que los patrones en los mercados laborales argentino y brasileño son similares a los del mercado de trabajo mexicano. En efecto, identifican otras similitudes entre estos mercados laborales. En primer lugar, tanto desempleo como informalidad son anticíclicas: ambas variables aumentan en fase de recesión y decrecen en fase de expansión. En segundo lugar, las transiciones de informalidad a formalidad y viceversa son procíclicas, en gran medida debido a la magnitud de las transiciones desde el empleo formal al empleo por cuenta propia. En un estudio sobre el mercado laboral en El Salvador, Funkhouser (1997) estimó la transición de acuerdo con las características de género. Descubrió que existía bastante movilidad entre los sectores formal e informal. De entre los trabajadores empleados originalmente de manera informal, un 7,3\% se cambió al empleo formal, mientras que la transición de empleo formal a informal fue de en torno al 4,7\%. Frente a esto, los porcentajes de trabajadoras fueron inferiores: un 2,1\% y un 3,3\% respectivamente.

Para el caso de México, Rodríguez-Oreggia (2007) estima la probabilidad de transición de empleo informal a formal y viceversa para diferentes períodos de tiempo. Concluye que la probabilidad de esta transición es mayor en el caso de transiciones de empleo informal a formal que en el sentido contrario. Alcaraz, Chiquiar y Salcedo (2015) utilizan un modelo de autoselección para estimar la prevalencia entre trabajadores voluntarios y no voluntarios. Concluyen que entre un $10 \%$ y un $20 \%$ de los trabajadores informales preferirían un trabajo formal, pero también que las barreras de acceso al empleo formal son estadísticamente significativas. Confirman la existencia de segmentación, con un cierto nivel de integración entre el empleo formal y el informal.

Hasta hace no mucho, el mercado laboral de México se consideraba muy rígido; Heckman y Pagés-Serra (2000) y Gil, Montenegro y Dömeland (2001), entre otros, argumentaban que estaba fuertemente regulado por normas que impedían la creación de empleo. La rigidez de la Ley Federal del Trabajo dificultaba y encarecía para las empresas los despidos de trabajadores en época de recesión, al tiempo que hacía que estas dudaran a la hora de realizar nuevas contrataciones en fase de expansión por los elevados costos, principalmente derivados del requisito de brindar beneficios sociales tales como seguro de gastos médicos, préstamos de vivienda o vacaciones pagadas, entre otros. Este tipo de rigidez del mercado explicaba por qué las variaciones en la producción solo se traducían en pequeñas variaciones en el desempleo, tal y como señala González Anaya (2002).

No obstante, estudios recientes reflejan los cambios significativos que ha experimentado el mercado laboral mexicano, especialmente por lo que respecta al gran crecimiento del empleo informal. La existencia de un gran mercado informal con una importante movilidad de la mano de obra entre empleo formal e informal sugiere que estamos ante un mercado laboral híbrido en el que las empresas del sector formal recurren cada vez más a diferentes sistemas de empleo con objeto de reducir sus costos laborales (Contreras, 2000; De la Garza, s/f).

Además, dada la magnitud del empleo informal y habida cuenta de las barreras de acceso al empleo formal, se podría esperar, por ejemplo, que un descenso en la producción llevara a las empresas a restringir sus planes de empleo ${ }^{9}$. A su vez, esto haría que los trabajadores aceptaran un puesto de trabajo informal o que quedaran desempleados. Del mismo modo, un incremento en la producción motivaría a las empresas a aumentar las contrataciones dentro de los sistemas flexibles que estuvieran a su disposición. El empleo informal se reduciría, al igual que el desempleo. Cabe destacar que, en ambos casos, los cambios en el desempleo serían menores que si no existiera el sector informal. Esto puede observarse en la elevada probabilidad de transición del empleo formal al informal, que impide que se produzca una transición mucho mayor del empleo formal al desempleo.

\footnotetext{
9 En algunos casos, las empresas reducen el número de empleados de tiempo completo y aumentan el número de empleados de tiempo parcial en el marco de un sistema de subcontratación.
} 


\section{Algunas observaciones acerca del coeficiente de Okun}

Desde la publicación del innovador artículo de Okun, la literatura sobre la relación entre crecimiento de la producción y variaciones en el desempleo ha aumentado considerablemente. Esta literatura respalda la validez empírica de la compensación entre estas dos variables, pese a que existen innumerables estudios que apuntan a que la magnitud del coeficiente de Okun varía significativamente en un determinado país (a lo largo del tiempo) y de un país a otro - véanse, por ejemplo, Silvapulle, Moosa y Silvapulle (2004), Lee (2000) o Harris y Silverstone (2001)-. Estos estudios suponen un cambio radical con respecto a documentos anteriores en los que se daba por hecho que el coeficiente era estable y confiable a lo largo del tiempo (Gordon, 1984).

El hecho de que el coeficiente no sea constante se ha atribuido a diferentes factores. Desde una perspectiva contable, varios autores han señalado que su magnitud depende de la evolución de variables como la tecnología, las horas de trabajo, la tasa de utilización del capital (Prachowny, 1993) y la sustitución de factores (Courtney, 1991). Otros estudios han indicado que el coeficiente también es sensible a la especificación de modelos, incluida la forma del modelo (estática frente a dinámica), y al método de suavizamiento utilizado para eliminar la no estacionariedad: modelo en primera diferencia frente a modelo de brechas (Crespo, 2003) ${ }^{10}$.

Una de las primeras investigaciones sobre la relación no lineal entre crecimiento de la producción y variación del desempleo fue la de Courtney (1991). Siguiendo con una larga tradición de investigación sobre los ciclos económicos en los Estados Unidos, Courtney encontró que el coeficiente de Okun dependía del estado del ciclo económico. En particular, llegó a la conclusión de que el efecto del crecimiento de la producción sobre la variación del desempleo era mayor en fases de contracción que en fases de expansión. Además, argumentó que la acumulación inadvertida de mano de obra puede ser un importante factor a la hora de determinar la dependencia del estado en la ley de Okun. En concreto, afirmó que el comportamiento asimétrico del empleo a lo largo del ciclo económico se explicaba por la sustitución entre las horas y el esfuerzo de la mano de obra.

A priori no podemos determinar si las variaciones de la producción tienen mayor impacto sobre las variaciones del desempleo en fases de expansión o de recesión. Existen dos explicaciones contradictorias. Por un lado, la perspectiva de que, cuando la economía empieza a contraerse, las empresas responden rápidamente con el despido de empleados. A medida que la recesión termina, ajustan la productividad, el número de horas trabajadas o ambas en lugar de recuperar el número de trabajadores, por miedo a que la recuperación no sea duradera (Jardin y Stephan, 2011). Este comportamiento asimétrico explicaría el hecho de que el crecimiento de la producción tenga un mayor efecto sobre el desempleo durante las fases de recesión que durante las fases de expansión. El punto de vista contrario pasa por que las empresas no quieren deshacerse de sus empleados en época de recesión por el elevado costo que ello implica (como consecuencia de la legislación laboral) y porque con ello perderían su inversión en la formación de los trabajadores. También se argumenta que estas empresas deberían contratar más trabajadores durante la recuperación porque en este período existen pocas restricciones institucionales. Desde esta perspectiva, se espera que el crecimiento de la producción tenga un mayor impacto sobre el desempleo durante las fases de expansión que durante las fases de recesión.

\footnotetext{
${ }^{10}$ En el modelo en primera diferencia, la producción y el desempleo vienen expresados en primera diferencia (tasas de crecimiento), mientras que, en el modelo de brechas, se consideran desviaciones de la tendencia a largo plazo. En este último, se plantea una nueva cuestión: qué filtro utilizar. Por ejemplo, en un análisis comparativo de economías desarrolladas, Lee (2000) evaluó la estabilidad y robustez de la ley, a partir de los cambios que se habían producido en las instituciones de los mercados laborales de muchos países europeos.
} 
En este estudio se llegó a varias conclusiones más. En primer lugar, a pesar de que los resultados fueran similares desde un punto de vista cualitativo, los autores encontraron importantes diferencias cuantitativas entre países. En segundo lugar, el modelo de brechas aportó pruebas más sólidas de que el coeficiente no se mantuvo constante a lo largo del tiempo en los distintos países que el modelo en primera diferencia. En tercer lugar, a principios de la década de 1970 se encontró un cambio estructural en la mayoría de los países europeos incluidos en el estudio, con excepción de Austria y, en el caso de los países no europeos, del Canadá. En cuarto y último lugar, el coeficiente fue mucho más bajo en la mayoría de los países europeos que en los Estados Unidos.

Harris y Silverstone (2001) ampliaron aún más el análisis de la asimetría en el caso de países clave de la Organización para la Cooperación y el Desarrollo Económicos (OCDE); para ello, estimaron los coeficientes a largo y corto plazo ${ }^{11}$. Concluyeron que el coeficiente a largo plazo se ubicaba entre $-0,39$ y - 0,5 y que el Japón y el Reino Unido presentaban valores atípicos. En cuanto al corto plazo, los resultados de su estudio indicaron que el desempleo se ajustaba al crecimiento de la producción de manera asimétrica; específicamente, que se ajustaba del modo esperado durante la desaceleración del ciclo económico. No obtuvieron resultados confiables en lo que respecta a la respuesta de la producción a las variaciones en el desempleo. En términos de implicaciones políticas, una ley de Okun no lineal sugeriría la necesidad de una respuesta política asimétrica para reducir las fluctuaciones de la producción. En algunos casos bastarán variaciones en el desempleo, mientras que en otros será preciso recurrir a ajustes de precio para restablecer el equilibrio.

Crespo (2003) y Silvapulle, Moosa y Silvapulle (2004), entre otros, han concluido que los modelos no lineales explican la relación entre los componentes cíclicos de la producción y el desempleo mejor que los modelos lineales, con independencia de la técnica de filtrado que se utilice. En ambos estudios se concluye también que el impacto de la producción en el desempleo es más fuerte en fases de recesión que en fases de expansión ${ }^{12}$.

Los trabajos revisados hasta este momento se caracterizan por aplicar un enfoque determinista para estimar el coeficiente asimétrico de Okun en el sentido de que el paso de un régimen a otro es un evento exógeno y determinista. Sin embargo, desde principios de la década de 2000 esta visión se ha visto superada por un nuevo enfoque que no solo mide este cambio por la probabilidad de transición entre los dos estados, sino que plantea que el cambio de régimen venga motivado por la magnitud de la brecha de producción. Además, el modelo estima también el tamaño de la brecha de producción que es necesario para inducir el cambio de régimen.

\section{La informalidad y el coeficiente de Okun: el caso de México}

Existen pocos estudios que hayan estimado el coeficiente de Okun para México y no hay un consenso sobre su magnitud. Por ejemplo, Chavarín (2001) estimó que el coeficiente sería cercano a los cálculos originales de Okun para la economía de los Estados Unidos ${ }^{13}$. Sin embargo, González Anaya (2002)

\footnotetext{
${ }^{11}$ La muestra incluía a Alemania, Australia, el Canadá, los Estados Unidos, el Japón, Nueva Zelandia y el Reino Unido. Utilizaron la metodología de Engle-Granger para series cointegradas, en que el término de corrección del error se ajusta para incorporar la asimetría. Su procedimiento incluyó la estimación de los puntos de umbral que minimizaban la suma al cuadrado de los valores residuales a través de una búsqueda por cuadrícula.

${ }^{12}$ Crespo (2003), por ejemplo, aplica tanto el filtro Hodrick-Prescott (HP) a cada una de las series de manera individual como el modelo bivariado estructural de series de tiempo propuesto por Harvey (1989). Silvapulle, Moosa y Silvapulle (2004) utilizan también la metodología bivariada de Harvey para encontrar la tendencia de la serie.

${ }^{13}$ Chavarín (2001) estimó que una variación del desempleo de 1 punto porcentual provocaría una variación de aproximadamente 2,7 puntos porcentuales en la producción. Por el contrario, una variación de la producción de 1 punto porcentual estaría asociada a una variación de 0,3 puntos porcentuales en el desempleo en sentido opuesto.
} 
e Islas y Cortez (2013) ${ }^{14}$ llegan a coeficientes mucho menores. Estos coeficientes menores son intrigantes, ya que la creciente flexibilidad del mercado laboral mexicano desde mediados de la década de 1990 parecería indicar que el efecto del crecimiento de la producción sobre el desempleo debería haber sido mucho mayor.

No obstante, no está claro que una mayor flexibilidad laboral se refleje necesariamente en mayores fluctuaciones en el empleo. Existen dos respuestas a la pregunta acerca de los posibles efectos de las fluctuaciones de la producción sobre las fluctuaciones del desempleo en el caso de mercados laborales más flexibles. Si esta flexibilidad hace referencia a las condiciones del empleo, es decir, si las empresas pueden contratar y despedir a sus empleados de acuerdo con sus necesidades de producción, entonces la respuesta es que las fluctuaciones de la producción sí tendrían un mayor efecto sobre las fluctuaciones del desempleo. Sin embargo, si la flexibilidad hace referencia a la capacidad de contratar trabajadores en el marco de programas flexibles de empleo, entonces las fluctuaciones de la producción tendrían un impacto reducido sobre las fluctuaciones del desempleo, si bien promoverían una mayor movilidad de los trabajadores entre los distintos tipos de empleo. La tasa de desempleo seguiría viéndose afectada por las fluctuaciones de la producción, pero en menor medida ${ }^{15}$.

Aquí es cuando el sector informal entra en escena. Se ha reconocido que varios de estos tipos de trabajos son realmente trabajos informales, en el sentido de que no aportan los beneficios sociales básicos que caracterizan al empleo formal. Si se tiene también en cuenta el empleo en empresas informales, la producción cíclica debería tener un efecto aún menor sobre el desempleo cíclico. Las pruebas presentadas en la sección II ponen claramente en evidencia la importante escala de transición del empleo formal al informal (y viceversa). Así pues, cuando existe un sector informal grande, el efecto de la producción cíclica sobre el empleo cíclico debería ser bastante reducido; este hallazgo ya fue señalado por Agénor y Aizenman (1999) y por Dell'Anno y Solomon (2008).

Tal y como se ha indicado en la sección II, existen varias definiciones de informalidad. De aquí en adelante, se considerará que el empleo informal (o empleo en el sector informal) incluye a las personas que trabajan en empresas informales. Las empresas informales son aquellas que no están inscritas ante ningún organismo gubernamental. Puesto que el propósito del presente estudio es analizar cómo el empleo informal puede afectar a la relación entre los componentes a corto plazo de desempleo y producción, no se hará distinción entre los distintos tipos de empleo informal.

En el cuadro 1 se presenta una correlación cruzada entre las tasas de crecimiento del producto interno bruto (PIB), el empleo informal y el desempleo.

En el cuadro 1 se muestran las correlaciones cruzadas entre el crecimiento del PIB y el empleo informal (primera fila), entre el crecimiento del PIB y el desempleo (segunda fila) y entre el crecimiento del desempleo y el empleo informal (tercera fila). Los resultados indican que el empleo informal lidera el ciclo y es procíclico. Cuando se tiene en cuenta el empleo informal desfasado, su relación con la producción cae en valores negativos, lo cual significa que se vuelve anticíclico. Tal y como cabría esperar, el desempleo es anticíclico y contemporáneo. Los resultados indican además que las tasas de crecimiento del desempleo y del desempleo informal son contemporáneas y tienen una relación negativa.

Las medidas tradicionales del coeficiente de Okun para la relación entre desempleo y producción muestran que son contemporáneas. Los resultados presentados en el cuadro 1 parecen confirmarlo, pero también sugieren que el empleo informal comienza a señalar una recuperación o ralentización algunos trimestres antes de que se manifieste en la producción. También sugieren que la relación no se mantiene constante en el tiempo, como se puede observar por el signo y la magnitud del coeficiente de correlación en el rezago 5.

\footnotetext{
${ }^{14}$ Islas y Cortez (2013) estiman que una variación de la producción de 1 punto porcentual está asociada a una variación en el desempleo de -0,5 puntos porcentuales, mientras que una variación del desempleo de 1 punto porcentual se asociaría a una variación en la producción de -1,66 puntos porcentuales.

15 Gong, van Soest y Villagomez (2004) encontraron pruebas de que la probabilidad de transición al desempleo desde un empleo tanto formal como informal era mayor en períodos de recesión que en períodos de expansión.
} 


\section{Cuadro 1}

México: correlación cruzada entre crecimiento del PIB, empleo informal y desempleo, primer trimestre de 1993 a segundo trimestre de 2015

\begin{tabular}{|c|c|c|c|c|c|c|c|c|c|c|c|c|c|}
\hline & -6 & -5 & -4 & -3 & -2 & -1 & 0 & 1 & 2 & 3 & 4 & 5 & 6 \\
\hline (PIB,enf a $)$ & 0,009 & 0,211 & 0,149 & 0,012 & 0,104 & $-0,059$ & $-0,102$ & $-0,008$ & $-0,197$ & $-0,097$ & 0,124 & 0,028 & 0,014 \\
\hline$\left(P I B, \mu^{p}\right)$ & 0,053 & 0,032 & $-0,069$ & 0,093 & 0,004 & $-0,143$ & $-0,375$ & $-0,307$ & $-0,082$ & 0,058 & $-0,070$ & 0,054 & 0,037 \\
\hline$(\mu, e n f)$ & $-0,050$ & 0,048 & $-0,019$ & $-0,121$ & 0,037 & $-0,050$ & $-0,315$ & 0,182 & 0,022 & 0,033 & 0,077 & 0,017 & 0,074 \\
\hline
\end{tabular}

Fuente: Elaboración propia, sobre la base de datos de la Encuesta Nacional de Empleo Urbano (ENEU) y la Encuesta Nacional de Ocupación y Empleo (ENOE).

a Empleo informal.

b Desempleo.

Este último punto va en la misma línea que la conclusión de Oliveira (2002), según la cual el ciclo económico de México es asimétrico y sus recesiones son más breves e intensas que sus expansiones. Por lo tanto, argumentamos que la relación entre las tasas de crecimiento del desempleo y de la producción depende del estado en que se encuentre la economía y, específicamente, que esta relación es más fuerte en fases de recesión que en fases de expansión.

Ahora, con objeto de valorar el impacto del empleo informal sobre la probabilidad de transición de un régimen a otro, definiremos el régimen en que se encuentra la economía por su tasa de desempleo. Se considerará que la economía está en expansión cuando el desempleo actual sea inferior a la tendencia de largo plazo, y se considerará que está en recesión cuando se encuentre por encima de la misma.

En la siguiente sección se proporcionan detalles sobre la probabilidad de transición de un régimen a otro, así como sobre la técnica de estimación.

\section{Metodología}

\section{Modelos empíricos}

Estimamos tres modelos para medir la compensación entre la producción cíclica y el desempleo cíclico. El modelo 1 asume una relación lineal, el modelo 2 es un modelo de régimen cambiante de Márkov con probabilidad de transición fija (PTF), y el modelo 3 es un modelo de probabilidad de transición variable en el tiempo que relaja la asunción de probabilidad de transición fija. Como explican Filardo (1994) y Diebold y Rudebusch (1999), el modelo de Márkov con probabilidad de transición variable en el tiempo (PTV) es más flexible que el modelo con probabilidad de transición fija. Reconoce cambios sistemáticos en las probabilidades de transición antes y después de los puntos de inflexión, captura una persistencia temporal más compleja y permite que la duración esperada varíe a lo largo del tiempo. En este contexto, los fundamentos económicos y las conmociones en materia de políticas pueden influir en las probabilidades de transición de un régimen a otro.

En primer lugar, consideramos el modelo tradicional de regresión lineal propuesto por Moosa (1997), al que denominaremos modelo 1. Es el siguiente:

$$
u_{t}^{c}=\alpha_{0}+\beta y_{t}^{c}+\sum_{i=1}^{p} \alpha_{j} u_{t-j}^{c}+\varepsilon_{t}, \varepsilon_{t} \sim N I D\left(0, \sigma^{2}\right)
$$

donde $u_{t}^{c}$ es el desempleo cíclico y $y_{t}^{c}$ la producción cíclica. Debe incluirse el desempleo cíclico desfasado para eliminar la autocorrelación. El coeficiente de Okun se mide a través del valor estimado de $\beta$, el coeficiente de impacto, tal que $\beta<0$. 
Además del modelo de regresión lineal, consideramos también el modelo con cambios de régimen de Márkov con probabilidad de transición fija (PTF) para caracterizar la especificación de dependencia de régimen de la ley de Okun, lo cual permite un efecto asimétrico de la producción cíclica sobre el desempleo cíclico. La idea general tras este tipo de modelos con cambios de régimen es que los parámetros de regresión dependen de una variable de régimen estocástica no observable $s_{t} \in\{1,2\}$. El proceso estocástico para generar el régimen no observable es una cadena ergódica de Márkov definida por la probabilidad de transición $p_{i j}=\operatorname{Pr}\left(s_{t+1}=j \mid s_{t}=i\right)$, donde $i, j=102$. La probabilidad de transición $p_{i j}$ indica la probabilidad de que el estado $i$ será seguido del estado $j$. La matriz de transición es

$$
P=\left[\begin{array}{ll}
p_{11} & p_{21} \\
p_{12} & p_{22}
\end{array}\right]
$$

donde $p_{11}$ es la probabilidad de permanecer en el régimen de expansión, definido como el resultado en que la tasa de desempleo se encuentra por debajo de su tendencia, mientras que $p_{22}$, por su parte, es la probabilidad de permanecer en el régimen recesivo, definido como la situación en que la tasa de desempleo se ubica por encima de la tendencia.

El modelo 2 es la especificación de la ley de Okun que depende del régimen en el tiempo, la cual permite un efecto asimétrico. Es como sigue:

$$
u_{t}^{c}=\alpha_{0 s_{t}}+\beta_{s_{t}} y_{t}^{c}+\sum_{i=1}^{p} \alpha_{j-1 s_{t}} u_{t-j}^{c}+\varepsilon_{t}, \varepsilon_{t} \sim N I D\left(0, \sigma_{s_{t}}^{2}\right)
$$

donde $\alpha_{j s_{t}}=\alpha_{j 1}, \beta_{s_{t}}=\beta_{1}, \sigma_{s_{t}}^{2}=\sigma_{1}^{2}$ si $s_{t}=1$, para $j=0,1, \ldots, p$, mientras $\alpha_{j s t}=\alpha_{j 2}, \beta_{s t}=\beta_{2}, \sigma_{s_{t}}^{2}=\sigma_{2}^{2}$ si $s_{t}=2$, para $j=0,1, \ldots, p$.

El modelo 2 presenta dos coeficientes, $\beta_{1}$ y $\beta_{2}$. En principio se espera que $\beta_{1}, \beta_{2}<0$, con un desempleo cíclico que responde de manera negativa a la producción cíclica en regímenes tanto expansivos como recesivos.

Consideramos, por último, el modelo 3, que permite probabilidades de transición variables en el tiempo. Para las variables de información en $z_{t}$ elegimos la tasa de empleo informal, puesto que damos por sentado que esta ha sido la principal causa de las variaciones en la tasa de desempleo (véase la sección IV). En este sentido, nos apartamos de otros estudios que han recurrido a la brecha de producción o la utilización de capacidad como variable principal en la probabilidad de transición variable en el tiempo. Así pues, el modelo 3 considera las siguientes probabilidades de transición variables en el tiempo:

$$
\begin{aligned}
& P\left[s_{t}=1 \mid s_{t-1}=1, \underline{z}_{t-1} ; \underline{\delta}_{1}\right]=p\left(\underline{z}_{t-1}\right)=\Phi\left(\underline{z}_{t-1}^{\prime} \underline{\delta}_{1}\right) \\
& P\left[s_{t}=2 s_{t-1}=2, \underline{z}_{t-1} ; \underline{\delta}_{2}\right]=q\left(\underline{z}_{t-1}\right)=\Phi\left(\underline{z}_{t-1}^{\prime} \underline{\delta}_{2}\right)
\end{aligned}
$$

donde $\Phi($.$) hace referencia a la función de densidad acumulada de la distribución normal$ estándar que evoluciona como función de $\underline{z}_{t-1}^{\prime} \underline{\delta}_{i}, i=1,2$, donde el vector condicionante $(m x 1)$ $\underline{\underline{z}}_{t-1}^{\prime}=\left(1, \Delta e_{i n f_{t-1}}, \Delta e_{i n f} f_{t-2}, \ldots, \Delta e_{i n f} t_{t-m-1}\right), \quad \underline{\delta}_{i}{ }^{\prime}=\left(\delta_{i 0}, \delta_{i 1}, \ldots, \delta_{i, m-1}\right), i=1,2 \quad$ y $\quad \Delta e_{i n f} t$ denota la primera diferencia de la tasa de empleo informal. El proceso estocástico de dos puntos en $s_{t}$ se puede resumir a través de la matriz de transición 


$$
P\left[s_{t}=1 \mid s_{t-1}=1, \underline{z}_{t-1} ; \underline{\delta}_{i}\right]=\left[\begin{array}{cc}
p\left(\underline{z}_{t-1}\right) & 1-p\left(\underline{z}_{t-1}\right) \\
1-q\left(\underline{z}_{t-1}\right) & q\left(\underline{z}_{t-1}\right)
\end{array}\right]
$$

en la que el historial del estado del desempleo informal se encuentra en $\underline{z}_{t-1}$.

En el modelo de cambio de régimen de Márkov con probabilidad de transición variable en el tiempo, las probabilidades de transición pueden variar con el estado del empleo informal (al alza y a la baja). Las probabilidades en el modelo 3 reflejan la duración de la relación de Okun, expresadas como una especificación dependiente del régimen. Para la prueba de la especificación del modelo, seguimos a Engel y Hamilton (1990) y probamos la siguiente hipótesis:

$$
H_{0}^{s s}: \alpha_{j 1}=\alpha_{j 2}, \beta_{1}=\beta_{2}, \sigma_{1}^{2} \neq \sigma_{2}^{2} ; j=0,1,2, \ldots, p
$$

Si no se puede rechazar $H_{0}^{s s}$, la implicación es que el verdadero proceso de generación de datos deriva de un único estado y no de dos estados. La prueba estadística para la hipótesis $H_{0}^{s S}$ es la prueba de Wald, y sigue una distribución $\chi_{(v)}^{2}$. Si dejamos $\sigma_{1}^{2}=\sigma_{2}^{2}$, la prueba de $H_{0}^{s S}$ presenta el llamado "problema de parámetros indeseables", es decir: los parámetros $p_{11}$ y $p_{22}$ no están identificados.

Para estimar los modelos 1 a 3, precisamos de series de tiempo para los componentes no observados $u_{t}^{c}$ y $y_{t}^{c}$. Se pueden aplicar distintas metodologías para extraer los componentes cíclicos, como por ejemplo considerando modelos de series de tiempo univariados o múltiples. Aquí nos concentramos en un enfoque de serie temporal bivariado que asume que el vector de series de tiempo observado está formado por un vector no observado de tendencias más un vector de ciclos, y considera la correlación entre las componentes cíclicas de la producción y el desempleo. Laxton y Tetlow (1992) presentan una revisión histórica de los métodos de estimación para el producto potencial e informan que, desde la década de 1980, se ha venido recurriendo básicamente a dos enfoques: i) enfoques estructurales basados en un modelo económico estructural, como es el caso de Ford y Rose (1989) y de Adams y Coe (1992) y ii) enfoques estocásticos como los subyacentes en el filtro desarrollado por Hodrick y Prescott (1981 y 1997). Laxton y Tetlow (1992) combinaron estos enfoques y propusieron una técnica semiestructural conocida como filtro multivariado de Hodrick-Prescott (HPMV) -véanse, por ejemplo, Boone (2000) y Chagny y Lemoine (2002) - . No obstante, el HPMV no es un verdadero filtro multivariado, sino más bien un filtro de series de tiempo múltiples. La diferencia es similar a la existente entre una regresión múltiple, donde solo existe una variable dependiente que se desea explicar por medio de varias variables independientes, y una regresión multivariada, donde se pretende explicar de manera simultánea varias variables dependientes a través de una o más variables independientes.

Aquí se utiliza una técnica semiestructural diferente basada en un método de filtrado de series de tiempo múltiples que fue propuesto por Guerrero, Islas y Ramírez (2017) y que se conoce como filtro bivariado de Hodrick-Prescott (BHP). Se trata de un nuevo método que se puede usar para extraer un par de tendencias que pudieran compartir comportamientos dinámicos similares. Una ventaja de este método es que solo emplea los dos primeros momentos muestrales de las variables en cuestión. Una segunda ventaja importante es que proporciona una forma de decidir el valor del parámetro de suavizado que produce el porcentaje de suavidad deseado para las tendencias. Una tercera ventaja es que tiene en cuenta la simultaneidad en la estimación, corrigiendo así probables sesgos. Es posible consultar más detalles sobre la metodología aplicada en el apéndice técnico disponible previa solicitud a los autores. 


\section{Los datos}

Las variables clave son producción, desempleo y empleo informal. Las cifras de PIB de México son las calculadas trimestralmente por el Instituto Nacional de Estadística y Geografía (INEGI) en pesos reales, tomando 2008 como año base. La serie de desempleo es la tasa de desempleo alternativa estimada según el método aplicado por la Oficina de Estadísticas Laborales de los Estados Unidos ${ }^{16}$. Las series laborales (desempleo y empleo informal) proceden de dos fuentes: la Encuesta Nacional de Empleo Urbano (ENEU) y la Encuesta Nacional de Ocupación y Empleo (ENOE).

Tanto la tasa de desempleo como la tasa del sector informal son estimaciones a partir de las 11 ciudades más grandes de México ${ }^{17}$. Solo se han seleccionado estas ciudades por ser las únicas que aparecen en ambas encuestas (ENEU y ENOE) y que, por tanto, podían emplearse para elaborar una serie de tiempo. Se han considerado trabajadores de entre 16 y 75 años. Todos los datos son trimestrales y están corregidos por la estacionalidad; cubren el período desde el primer trimestre de 1993 hasta el segundo trimestre de 2015. Los empleados del sector informal se definen como aquellos empleados que trabajan en empresas no registradas ${ }^{18}$.

En el gráfico 1 se describe el comportamiento de la estimación conjunta de los componentes cíclicos de producción y desempleo; para su extracción se ha utilizado la metodología propuesta por Guerrero, Islas y Ramírez (2017). En él se muestra que el comportamiento de los componentes cíclicos es coherente con la teoría económica de la ley de Okun, lo cual implica que el desempleo cíclico solo es negativo en caso de que la producción cíclica sea positiva, y viceversa.

\section{Gráfico 1}

México: estimaciones de producción y desempleo cíclicos, filtro bivariado de Hodrick-Prescott con un 90\% de suavidad, primer trimestre de 1990

a primer trimestre de 2015

\section{A. Componente cíclico del desempleo}

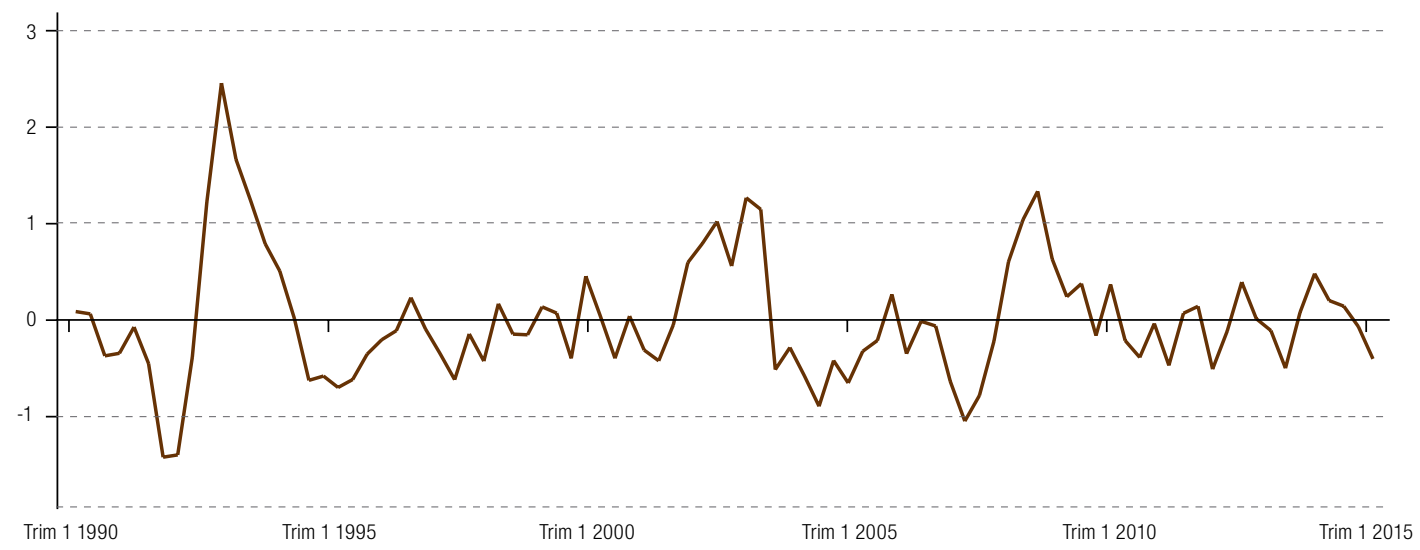

16 Véase una discusión sobre las diferencias más importantes entre la metodología utilizada por el INEGl de México y la aplicada por la Oficina de Estadísticas Laborales a la hora de estimar el desempleo en Fleck y Sorrentino (1994) y Martin (2000). Tradicionalmente, la metodología del INEGI ha venido subestimado gravemente la tasa de desempleo y, si bien ahora se han ajustado algunos aspectos, siguen existiendo elementos que favorecen la tasa de desempleo de México con respecto a la de la economía de los Estados Unidos.

${ }^{17}$ Las ciudades consideradas para el análisis son Chihuahua, Guadalajara, León, Mérida, Ciudad de México, Monterrey, Puebla, San Luis Potosí, Tampico, Tijuana y Veracruz.

${ }^{18}$ Cabe indicar que esta definición de informalidad difiere de la definición de empleo informal, que hace referencia al empleo que no dispone de prestaciones sociales, pero que en algunos casos puede presentarse en empresas formales. 
Gráfico 1 (conclusión)

\section{B. Componente cíclico de la producción}

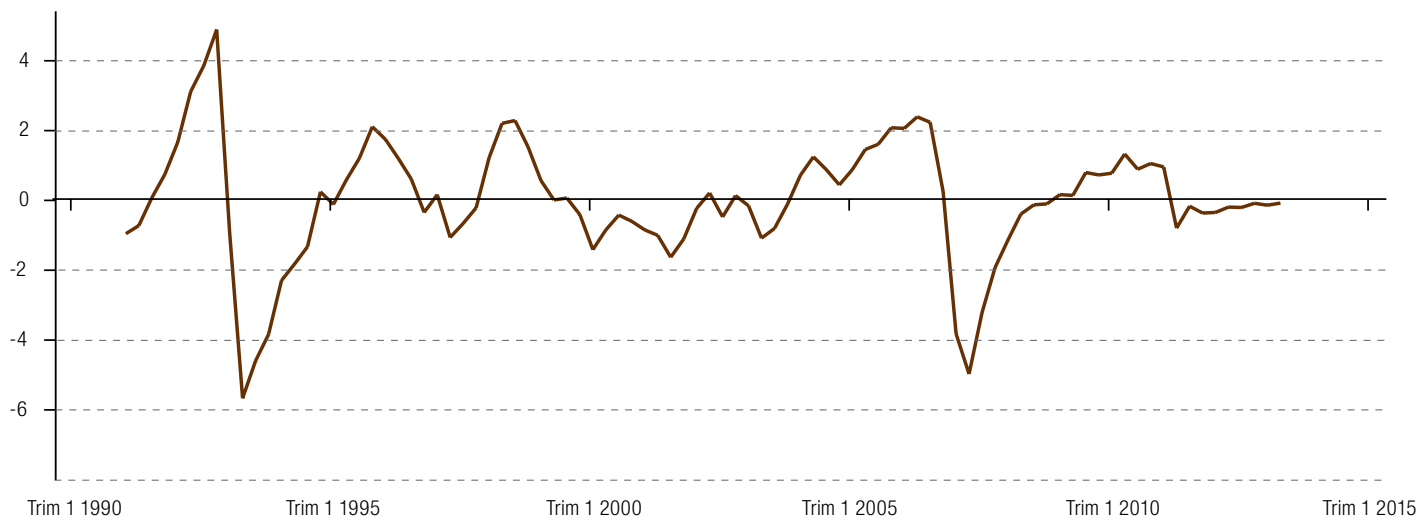

Fuente: Elaboración propia.

\section{Resultados empíricos}

Tras obtener los componentes cíclicos $u_{t}^{c}$ y $y_{t}^{c}$, se procedió a estimar el modelo lineal y los no lineales. En el cuadro 2 se presentan los resultados para los tres modelos.

El número de rezagos del componente autorregresivo del desempleo cíclico, $p$, se escogió a través del criterio de información de Akaike. Partiendo de un máximo de seis rezagos, se concluyó que se aceptaba la inclusión de un rezago de $u^{c}$ para el modelo 1 , cinco para el modelo 2 y seis para el modelo 3 (junto con un rezago para $\Delta e_{i n f_{t}}$ en el modelo PTV).

Cuadro 2

Resultados estimados de los modelos 1,2 y 3

\begin{tabular}{|c|c|c|c|c|c|}
\hline \multicolumn{2}{|c|}{ Modelo 1} & \multicolumn{2}{|c|}{ Modelo 2} & \multicolumn{2}{|c|}{ Modelo 3} \\
\hline Parámetro & Estimación & Parámetro & Estimación & Parámetro & Estimación \\
\hline$\alpha_{0}$ & $\begin{array}{l}-0,0010 \\
(0,0430)\end{array}$ & $\alpha_{01}$ & $\begin{array}{l}-0,0374 \\
(0,0395)\end{array}$ & $\alpha_{01}$ & $\begin{array}{r}-0,0567^{\star \star} \\
(0,0288)\end{array}$ \\
\hline$\beta_{1}$ & $\begin{array}{r}-0,1806^{\star \star *} \\
(0,0314)\end{array}$ & $\beta_{1}$ & $\begin{array}{r}-0,1207^{\star \star \star} \\
(0,0377)\end{array}$ & $\beta_{1}$ & $\begin{array}{r}-0,1092^{* \star \star} \\
(0,0295)\end{array}$ \\
\hline \multirow[t]{10}{*}{$\alpha_{1}$} & $\begin{array}{r}0,4404^{\star \star \star} \\
(0,0623)\end{array}$ & $\alpha_{11}$ & $\begin{array}{r}0,4059^{\star \star \star} \\
(0,0924)\end{array}$ & $\alpha_{11}$ & $\begin{array}{r}0,3875^{\star \star \star} \\
(0,0220)\end{array}$ \\
\hline & & $\alpha_{21}$ & $\begin{array}{l}0,0335 \\
(0,186)\end{array}$ & $\alpha_{21}$ & $\begin{array}{l}-0,0601 \\
(0,0106)\end{array}$ \\
\hline & & $\alpha_{31}$ & $\begin{array}{r}0,0894 \\
(0,1025)\end{array}$ & $\alpha_{31}$ & $\begin{array}{r}0,2070^{\star *} \\
(0,0971)\end{array}$ \\
\hline & & $\alpha_{41}$ & $\begin{array}{r}-0,2414^{\star \star} \\
(0,0876)\end{array}$ & $\alpha_{41}$ & $\begin{array}{r}-0,3751^{\star \star \star} \\
(0,1009)\end{array}$ \\
\hline & & $\alpha_{51}$ & $\begin{array}{r}0,0002 \\
(0,0832)\end{array}$ & $\alpha_{51}$ & $\begin{array}{r}0,1523 \\
(0,1119)\end{array}$ \\
\hline & & $\alpha_{02}$ & $\begin{array}{r}0,2259^{\star *} \\
(0,0923)\end{array}$ & $\alpha_{61}$ & $\begin{array}{r}-0,1543^{*} \\
(0,0828)\end{array}$ \\
\hline & & $\beta_{2}$ & $\begin{array}{r}-0,3117^{\star \star \star} \\
(0,0152)\end{array}$ & $\alpha_{20}$ & $\begin{array}{l}0,2307^{*} \\
(0,0932)\end{array}$ \\
\hline & & $\alpha_{12}$ & $\begin{array}{r}0,7537^{\star \star *} \\
(0,1391)\end{array}$ & $\beta_{2}$ & $\begin{array}{r}-0,2606^{\star \star \star} \\
(0,0369)\end{array}$ \\
\hline & & $\alpha_{22}$ & $\begin{array}{c}-0,4835^{\star} \\
(0,2534)\end{array}$ & $\alpha_{21}$ & $\begin{array}{r}0,5920^{* \star *} \\
(0,1572)\end{array}$ \\
\hline & & $\alpha_{32}$ & $\begin{array}{r}1,2359^{\star \star \star} \\
(0,2754)\end{array}$ & $\alpha_{22}$ & $\begin{array}{r}0,0803 \\
(0,2241)\end{array}$ \\
\hline
\end{tabular}


Cuadro 2 (conclusión)

\begin{tabular}{|c|c|c|c|c|c|}
\hline \multicolumn{2}{|c|}{ Modelo 1} & \multicolumn{2}{|c|}{ Modelo 2} & \multicolumn{2}{|c|}{ Modelo 3} \\
\hline \multirow{11}{*}{ Parámetro } & Estimación & Parámetro & Estimación & Parámetro & Estimación \\
\hline & & $\alpha_{42}$ & $\begin{array}{r}-0,7215^{\star \star} \\
(0,2417)\end{array}$ & $\alpha_{32}$ & $\begin{array}{r}0,0503 \\
(0,1992)\end{array}$ \\
\hline & & $\alpha_{52}$ & $\begin{array}{r}-0,9283^{\star \star \star} \\
(0,2742)\end{array}$ & $\alpha_{42}$ & $\begin{array}{r}0,1812 \\
(0,2021)\end{array}$ \\
\hline & & $\sigma_{11}^{2}$ & $\begin{array}{c}0,0811^{\star *} \\
(0,0166)\end{array}$ & $\alpha_{52}$ & $\begin{array}{r}-0,3859^{*} \\
(0,2224)\end{array}$ \\
\hline & & $\sigma_{12}^{2}$ & $\begin{array}{r}0,0445^{\star *} \\
(0,0219)\end{array}$ & $\alpha_{62}$ & $\begin{array}{l}-0,0918 \\
(0,1474)\end{array}$ \\
\hline & & $p_{11}$ & $\begin{array}{r}0,9054^{\star \star *} \\
(0,0650)\end{array}$ & $\sigma_{11}^{2}$ & $\begin{array}{r}0,0813^{\star * *} \\
(0,0155)\end{array}$ \\
\hline & & $p_{12}$ & $\begin{array}{l}0,4650^{*} \\
(0,2814)\end{array}$ & $\sigma_{12}^{2}$ & $\begin{array}{c}0,0756^{* *} \\
(0,0242)\end{array}$ \\
\hline & & & & $\delta_{10}$ & $\begin{array}{r}2,8524^{\star \star} \\
(0,5551)\end{array}$ \\
\hline & & & & $\delta_{11}$ & $\begin{array}{l}2,4859^{*} \\
(1,4212)\end{array}$ \\
\hline & & & & $\delta_{20}$ & $\begin{array}{r}3,3282^{* *} \\
(1,5971)\end{array}$ \\
\hline & & & & $\delta_{21}$ & $\begin{array}{r}-4,7561^{*} \\
(2,8266)\end{array}$ \\
\hline \multicolumn{2}{|c|}{$\log L-40,0831$} & \multicolumn{2}{|c|}{$\log L-26,5087$} & \multicolumn{2}{|c|}{$\log L-22,3847$} \\
\hline
\end{tabular}

Prueba de selección del modelo: prueba de razón de verosimilitud

Modelo 1 frente a modelo 2: 27,148 ${ }^{\star \star \star}$. Modelo 2 frente a modelo 3: 8,2574*

Prueba de especificación del modelo $2: H_{0}^{s s}: \alpha_{j 1}=\alpha_{j 2}, \beta_{1}=\beta_{2}, j=0,1,2, \ldots, 5: 62,372^{* * *}$

Prueba de especificación del modelo 3: $H_{0}^{s s}: \alpha_{j 1}=\alpha_{j 2}, \beta_{1}=\beta_{2}, j j=0,1,2, \ldots, 6: 44,774 * * *$

Prueba de asimetría: modelo $2 H_{0}: \beta_{1}=\beta_{2} 15,923 * * *$. Modelo $3 H_{0}: \beta_{1}=\beta_{2} 11,952 * * *$

Fuente: Elaboración propia.

Notas: Los valores entre paréntesis son errores estándar. ${ }^{* \star *},{ }^{* \star} \mathrm{y}$ * representan niveles de significación del $1 \%$, el $5 \%$ y el $10 \%$, respectivamente.

Puesto que para este artículo se han considerado tres modelos diferentes, la prueba de razón de verosimilitud se utilizó en primer lugar para la selección de modelos y sus resultados se resumen en el cuadro 2. La estadística de la prueba de razón de verosimilitud sugiere que el modelo 2 (la especificación de la ley de Okun dependiente del régimen y con probabilidad de transición fija) es preferible al modelo 1 (la especificación lineal de la ley de Okun). Esto aporta pruebas sobre el carácter no lineal de la ley de Okun para México. Los resultados indican además que la tasa de desempleo cíclica media es menor en regímenes expansivos que en regímenes recesivos $(-0,0374<0,2259)$. Como ya se ha mencionado, los regímenes expansivos y recesivos se definen en función de que el desempleo se encuentre por debajo o por encima de su tendencia, respectivamente. En ambos regímenes, los dos coeficientes de Okun dependientes del estado $\left(\beta_{1}, \beta_{2}\right)$ son negativos y significativos en el nivel del $1 \%$. Tras la realización de pruebas adicionales se rechaza la hipótesis nula $H_{0}: \beta_{1}=\beta_{2}$, un resultado que apoya la existencia de un coeficiente de Okun asimétrico. El desempleo cíclico es más sensible a la producción cíclica contemporánea cuando se encuentra en un régimen recesivo. Los resultados indican que un descenso del $1 \%$ en la producción cíclica va acompañado de un aumento de aproximadamente el $0,31 \%$ en el desempleo si el sistema se encuentra en un régimen recesivo, mientras que un incremento del 1\% en la producción cíclica reduce el desempleo aproximadamente un $0,12 \%$ cuando el sistema se encuentra en un régimen expansivo.

Además, la probabilidad $p_{11}$ de permanecer en un régimen expansivo en el momento $(t)$, dado que la tasa de desempleo se encontraba en el mismo régimen en el momento $(t-1)$, es de 0,90. La probabilidad $p_{22}$ de encontrarse en un régimen recesivo en el momento $(t)$, dado que la tasa de 
desempleo se encontraba en el mismo régimen en el momento $(t-1)$, es de 0,53 , menor que $p_{11}$. Estos valores de las probabilidades de transición indican que, si la tasa de desempleo se encuentra en un régimen expansivo, es más probable que se mantenga en dicho régimen a que cambie a uno recesivo. Por otra parte, en el cuadro 2 se muestra que la probabilidad de cambiar de un régimen expansivo a uno recesivo es de casi 0,095 , mientras que la probabilidad de cambiar de un régimen recesivo a uno expansivo es cercana a 0,47; esto indica que los cambios de régimen recesivo a expansivo son más probables que los cambios de régimen expansivo a recesivo. La duración esperada de un régimen $j$ se define como $1 /\left(1-p_{j j}\right)$. A partir de este resultado, encontramos que la longitud media de un régimen expansivo se situó en dos años y medio, mientras que la longitud esperada de un régimen recesivo fue de aproximadamente medio año.

Ahora estimaremos el modelo en función de las probabilidades de transición endógenas. A diferencia de otros artículos que utilizan las variaciones en la producción, el presente estudio permite que sea la tasa de empleo informal la que explique la evolución de dichas probabilidades. Tal y como se explicó en la sección IV, se considera que el empleo informal es una de las causas principales de variaciones en la tasa de desempleo.

La prueba de razón de verosimilitud que compara el modelo de probabilidad de transición variable en el tiempo con el modelo de probabilidad de transición fija rechaza la hipótesis nula de probabilidades de transición fijas en favor de un modelo con probabilidades de transición variables en el tiempo. Sobre la base de estas pruebas, podemos concluir que el modelo con probabilidades de transición endógenas es el más indicado para explicar la relación entre los componentes cíclicos del desempleo y de la producción.

Las estimaciones del modelo con probabilidad de transición variable en el tiempo, que también se muestran en el cuadro 2, validan la existencia de dos estados distintos de la tasa de desempleo: un régimen expansivo con desempleo cíclico medio negativo $(-0,0567)$ y un régimen recesivo con desempleo cíclico medio positivo $(0,2307)$. En este caso, los valores del desempleo cíclico medio en regímenes expansivos y recesivos son similares a los encontrados para el caso del modelo con probabilidad de transición fija.

Se observa que el signo de la variable explicativa de las probabilidades de transición coincide con la intuición económica. De hecho, la probabilidad de permanecer en un régimen expansivo con desempleo por debajo de la tendencia aumenta cuando se produce un incremento en la tasa de empleo informal. Por otra parte, si la tasa de desempleo se encuentra en régimen recesivo, con desempleo por encima de la tendencia, entonces un incremento de la tasa de empleo informal reducirá la probabilidad de permanecer en este régimen.

Tal y como sucede en el modelo con probabilidad de transición fija, los dos coeficientes de Okun dependientes del estado $\left(\beta_{1}, \beta_{2}\right)$ son negativos y significativos a un nivel de significación del $1 \%$ para ambos regímenes. Tras la realización de pruebas adicionales se rechaza la hipótesis nula $H_{0}: \beta_{1}=\beta_{2}$, un resultado que apoya la existencia de un coeficiente de Okun asimétrico. El desempleo cíclico es más sensible al crecimiento económico contemporáneo cuando se encuentra en un régimen recesivo. Los resultados indican que un descenso del 1\% en la producción cíclica va acompañado de un aumento de aproximadamente el 0,26\% en el desempleo si la tasa de desempleo se encuentra en régimen recesivo, mientras que un incremento del $1 \%$ en la producción cíclica reduce el desempleo aproximadamente un $0,12 \%$ cuando el desempleo se encuentra en régimen expansivo. Nuestros resultados indican que, cuando se permite que el empleo informal explique la evolución de las probabilidades de transición, las estimaciones del coeficiente de Okun son menores que las estimaciones del modelo con probabilidad de transición fija. Esto corrobora nuestra afirmación de que el efecto de la producción cíclica sobre el empleo cíclico debe ser bastante reducido cuando existe un sector informal grande. 
En el gráfico 2 se muestra la probabilidad de transición variable en el tiempo suavizada para un régimen recesivo con desempleo por encima de la tendencia en cada fecha de la muestra, obtenida a partir del modelo de probabilidades endógenas. El momento en que la tasa de desempleo cambió de un régimen a otro se basa en $P\left(s_{t}=\operatorname{res} \mid u_{1}^{c}, \ldots, u_{T}^{c} ; \widehat{\Theta}\right) \geq 0,5$. El cambio entre regímenes suele ser repentino, completo y esporádico. La tasa de desempleo se encuentra en régimen expansivo la mayor parte del tiempo. En el gráfico 2 se presentan cuatro cambios de un régimen expansivo a uno recesivo durante el período muestral. El primero se produjo entre el cuarto trimestre de 1994 y el tercer trimestre de 1995, coincidiendo con la crisis financiera mexicana de 1994. El segundo tuvo lugar entre el segundo trimestre de 2003 y el primer trimestre de 2005: la tendencia al alza de la tasa de desempleo mexicana durante este período podría estar relacionada con la ralentización del país en ese momento, cuando la economía se estancó durante el segundo y el tercer trimestre de 2003, el segundo y el tercer trimestre de 2004 y el primer trimestre de 2005. El tercer cambio aparece en el segundo trimestre de 2008, al inicio de la crisis financiera mundial. El último cambio de un régimen expansivo a uno recesivo tuvo lugar en el primer trimestre de 2013 y podría estar relacionado con el desempeño de la economía de México. La economía creció un 1,1\% en 2013, lo cual refleja una fuerte caída desde la expansión del 3,9\% registrada en 2012 y marcó el desempeño más débil desde que en 2009 se produjera una profunda recesión.

Gráfico 2

Probabilidades de transición suavizadas de encontrarse en un régimen recesivo, modelo de probabilidades de transición variables en el tiempo (PTV)

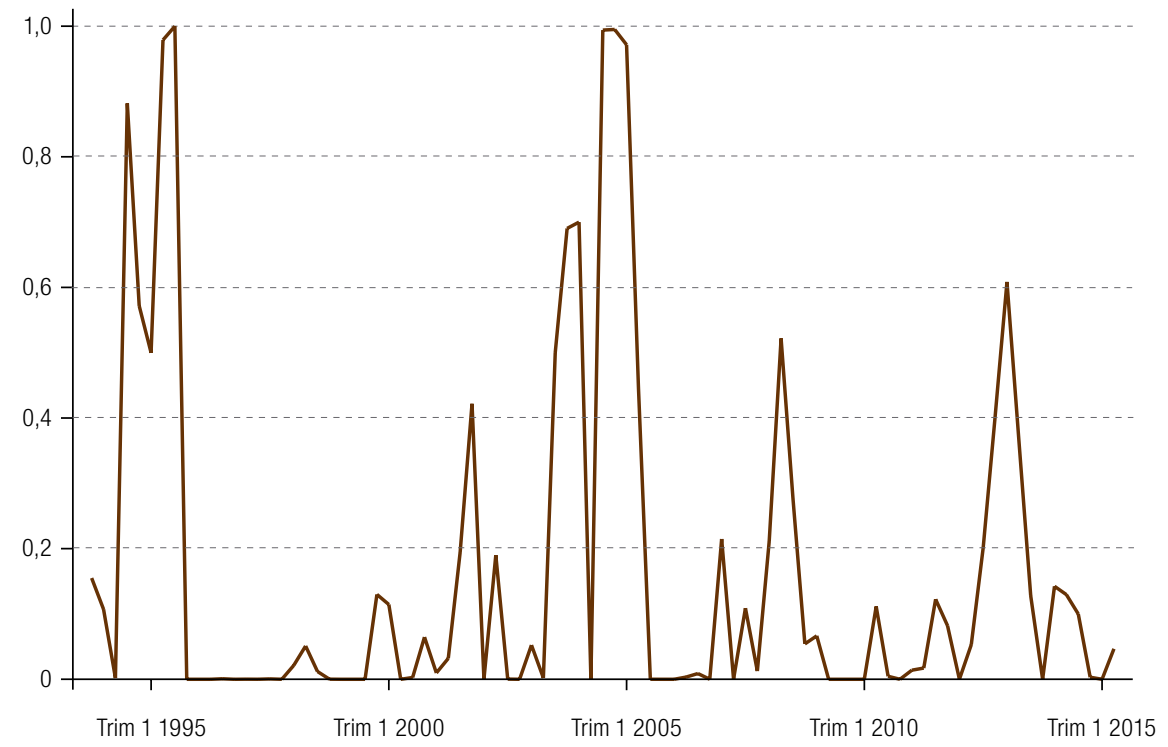

Fuente: Elaboración propia.

\section{Conclusiones}

En el presente artículo se evalúa el modo en que un gran sector informal influye en el impacto que las fluctuaciones de la producción tienen sobre las fluctuaciones del desempleo. También se considera la posibilidad de que esta influencia pueda cambiar a lo largo del ciclo económico de México. Se estimó una especificación no lineal de la relación entre desempleo cíclico y producción cíclica para el caso de la economía de México. Se empleó un modelo de Márkov con cambio de régimen y con probabilidades de transición tanto fijas como variables en el tiempo para identificar la presencia de asimetrías en los distintos regímenes. En concreto, en el modelo de probabilidades endógenas 
se permitió que las probabilidades se vieran afectadas por la tasa de empleo informal, la cual se considera la principal causa de las variaciones en la tasa de desempleo.

Nuestras conclusiones pueden resumirse del siguiente modo. En primer lugar, corroboramos que las estimaciones previas del coeficiente de Okun son bastante bajas. Defendemos que este coeficiente bajo se explica en gran medida por la existencia de un sector informal grande con un elevado nivel de movilidad entre los sectores formal e informal. En segundo lugar, encontramos pruebas de un coeficiente de Okun no lineal en México. En concreto, nuestros resultados apoyan la existencia de un coeficiente de Okun dependiente del régimen, con un valor absoluto notablemente superior en las recesiones que en las expansiones. Esto implica que el desempleo cíclico es más sensible a las variaciones en la producción cíclica cuando se encuentra en un régimen recesivo. En tercer lugar, se puede rechazar la hipótesis de probabilidades de transición fijas en favor de las probabilidades de transición variables en el tiempo, lo que supone que es mejor un modelo con probabilidades de transición endógenas. En cuarto lugar, proponemos que el empleo informal afecta de manera significativa a la evolución de la tasa de desempleo. Concluimos que la probabilidad de permanecer en un régimen expansivo con desempleo inferior a la tendencia aumenta cuando se incrementa la tasa de empleo informal, mientras que cuando la tasa de desempleo se encuentra en régimen recesivo y se sitúa por encima de la tendencia, un incremento en la tasa de empleo informal reducirá la probabilidad de permanecer en este régimen.

\section{Bibliografía}

Adams, Ch. y D. T. Coe (1992), "A systems approach to estimating the natural rate of unemployment and potential output for the United States", The United States Economy: Performance and Issues, Y. Horiguchi (ed.), Washington, D.C., Fondo Monetario Internacional (FMI).

Agénor, P. R. y J. Aizenman (1999), "Macroeconomic adjustment with segmented labor markets", Journal of Development Economics, vol. 58, № 2, Amsterdam, Elsevier.

Alcaraz, C., D. Chiquiar y A. Salcedo (2015), "Informality and segmentation in the Mexican labor market", Documentos de Investigación, № 2015-25, Ciudad de México, Banco de México.

Ball, L. y otros (2016), "Does one law fit all? Cross-country evidence on Okun's law", documento presentado en el Global Labor Markets Workshop, París, 1 y 2 de septiembre.

Bhattacharya, P. (2007), "Informal sector, income inequality and economic development", CERT Discussion Papers, N ${ }^{\circ} 709$, Centre for Economic Reform and Transformation, septiembre.

Boone, L. (2000), "Comparing semi-structural methods to estimate unobserved variables: the HPMV and Kalman filters approaches", OECD Economics Department Working Papers, N2 240, París, OECD Publishing.

Bosch, M. y W. Maloney (2010), "Comparative analysis of labor market dynamics using Markov processes: an application to informality", Labour Economics, vol. 17, № 4, Amsterdam, Elsevier.

Calderón, A. (2000), "Job stability and labor mobility in urban Mexico: a study based on duration models and transition analysis", Research Network Working Paper, N ${ }^{\circ}$ R-419, Washington, D.C., Banco Interamericano de Desarrollo (BID).

Chagny, O. y M. Lemoine (2002), "The impact of the macroeconomic hypothesis on the estimation of the output gap using a multivariate Hodrick-Prescott filter: the case of the Euro area", documento presentado en el Colloquium on Modern Tools for Business Cycle Analysis (28 y 29 de noviembre).

Chavarín, R. (2001), "El costo del desempleo medido en producto. Una revisión empírica de la ley de Okun para México", El Trimestre Económico, vol. 68, № 270, Ciudad de México, Fondo de Cultura Económica, abril-junio.

Contreras, O. (2000), "Los estudios acerca de la flexibilidad laboral en México: algunas observaciones críticas", Estudios Sociológicos, vol. 18, № 3, Ciudad de México, El Colegio de México.

Courtney, H. (1991), "Okun's law and business cycle in the Beveridge curve and Okun's law: a re-examination of fundamental relationships in the United States", tesis, Cambridge, Massachusetts, Instituto de Tecnología de Massachusetts (MIT), inédito.

Crespo, J. (2003), "Okun's law revisited", Oxford Bulletin of Economics and Statistics, vol. 65, № 4, Wiley. 
De la Garza, E. (s/f), "La flexibilidad del trabajo en México", inédito [en línea] http://sgpwe.izt.uam.mx/pages/ egt/publicaciones/articulos/flexibilidad.pdf.

Dell'Anno, R. y O. H. Solomon (2008), "Shadow economy and unemployment rate in USA: is there a structural relationship? An empirical analysis", Applied Economics, vol. 40, № 19, Taylor \& Francis.

Diebold, F. y G. Rudebusch (1999), "Measuring business cycles: a modern perspective", Business Cycles: Durations, Dynamics and Forecasting, Princeton, Princeton University Press.

Engel, C. y J. D. Hamilton (1990), "Long swings in the dollar: are they in the data and do markets know it?", The American Economic Review, vol. 80, № 4, Nashville, Tennessee, American Economic Association.

Filardo, A. (1994), "Business-cycle phases and their transitional dynamics", Journal of Business and Economic Statistics, vol. 12, № 3, Taylor \& Francis.

Fleck, S. y C. Sorrentino (1994), "Employment and unemployment in Mexico's labor force", Monthly Labor Review, vol. 117, № 11, Washington, D.C., Oficina de Estadísticas Laborales.

Ford, R. y D. Rose (1989), "Estimates of the NAIRU using an extended Okun's law", Documento de Trabajo, № 89-3, Ottawa, Banco de Canadá.

Funkhouser, E. (1997), "Mobility and labor market segmentation: the urban labor market in El Salvador", Economic Development and Cultural Change, vol. 46, No 1, Chicago, The University of Chicago Press.

Galli, R. y D. Kucera (2003), "Informal employment in Latin America: movements over business cycle and the effects of worker rights", Documento para Discusión, № DP/145/2003, Ginebra, Instituto Internacional de Estudios Laborales (IIEL).

Gil, I., C. Montenegro y D. Dömeland (eds.) (2002), Crafting Labor Policy: Techniques and Lessons from Latin America, Washington, D.C., Banco Mundial.

Goldberg, P. y N. Pavcnick (2003), "The response of the informal sector to trade liberalization", NBER Working Paper, № 9443, Cambridge, Massachusetts, Oficina Nacional de Investigaciones Económicas.

Gong, X., A. van Soest y E. Villagomez (2004), "Mobility in the urban labor market: a panel data analysis for Mexico", Economic Development and Cultural Change, vol. 53, № 1, Chicago, The University of Chicago Press.

González Anaya, J. (2002), "Labor market flexibility in thirteen Latin American countries and the United States: revisiting and expanding Okun coefficients", Documento de Trabajo, № 136, Stanford, Universidad de Stanford, junio.

Gordon, R. J. (1984), "Unemployment and potential output in the 1980s", Brookings Papers on Economic Activity, $N^{\circ} 2$, Washington, D.C., Brookings Institution.

Guerrero, V. M., A. Islas y R. L. L. Ramírez (2017), "Trend estimation of multivariate time series with controlled smoothness", Communications in Statistics - Theory and Methods, vol. 46, № 13, Taylor \& Francis.

Harris, R. y B. Silverstone (2001), "Testing for asymmetry in Okun's law: a cross-country comparison", Economics Bulletin, vol. 5, № 2.

Harvey, A. C. (1989), Forecasting, Structural Time Series and the Kalman Filter, Cambridge, Cambridge University Press.

Heckman, J. y C. Pagés-Serra (2000), "The cost of job security regulation: evidence from Latin American labor markets", Economía, vol. 1, № 1, Washington, D.C., Brookings Institution Press.

Hodrick, R. J. y E. C. Prescott (1997), "Postwar U.S. business cycles: an empirical investigation”, Journal of Money Credit and Banking, vol. 29, № 1, Ohio, Ohio State University Press.

(1981), "Post-war U.S. business cycles: an empirical investigation", Discussion Paper, № 451, Evanston, Illinois, Northwestern University.

Islas, A. y W. W. Cortez (2013), "Relaciones dinámicas del producto y el empleo en México: una evaluación de sus components permanentes y transitorios", Revista CEPAL, No 111 (LC/G.2597-P), Santiago, Comisión Económica para América Latina y el Caribe (CEPAL).

Jardin, M. y G. Stephan (2011), "How Okun's law is non-linear in Europe: a semi-parametric approach", mayo [en línea] http://euroframe.org/files/user_upload/euroframe/docs/2011/EUROF11_Jardin_Stephan.pdf.

Laxton, D. y R. Tetlow (1992), "A simple multivariate filter for the measurement of potential output", Technical Report, N 59, Banco de Canadá.

Lee, J. (2000), "The robustness of Okun's law: evidence from OECD countries", Journal of Macroeconomics, vol. 22, No 2, Amsterdam, Elsevier.

Maloney, W. (1998), "Are labor markets in developing countries dualistic?", Policy Research Working Paper, $N^{\circ} 1941$, Washington, D.C., Banco Mundial.

Martin, G. (2000), "Employment and unemployment in Mexico in the 1990s", Monthly Labor Review, Washington, D.C., Oficina de Estadísticas Laborales, noviembre. 
Moosa, I. A. (1997), "A cross-country comparison of Okun's coefficient", Journal of Comparative Economics, vol. 24, No 3, Amsterdam, Elsevier.

Okun, A. M. (1962), "Potential GNP: its measurement and significance", Proceedings of the Business and Economics Statistics Section, Alexandria, American Statistical Association.

Oliveira, A. (2002), "Are Mexican business cycles asymmetrical?", IMF Working Paper, Nº2/150, Washington, D.C., Fondo Monetario Internacional (FMI), septiembre.

Prachowny, M. (1993), "Okun's law: theoretical foundations and revised estimates", The Review of Economics and Statistics, vol. 75, № 2, Cambridge, Massachusetts, The MIT Press.

Rodríguez-Oreggia, E. (2007), "The informal sector in Mexico: characteristics and dynamics", Perspectivas Sociales, vol. 9, № 1, Universidad Autónoma de Nuevo León.

Silvapulle, P., I. A. Moosa y M. J. Silvapulle (2004), "Asymmetry in Okun's law", The Canadian Journal of Economics, vol. 37, No 2, Wiley.

Tokman, V. (2011), "Informalidad en América Latina: balance y perspectivas de políticas", Realidad, Datos y Espacio. Revista Internacional de Estadística y Geografía, vol. 2, № 3, Aguascalientes, Instituto Nacional de Estadística y Geografía (INEGI).

Vuletin, G. (2008), "Measuring the informal economy in Latin America and the Caribbean", IMF Working Paper, $N^{\circ}$ 08/102, Washington, D.C., Fondo Monetario Internacional (FMI). 